\title{
Two target genes based multiple cross displacement amplification combined with a lateral flow biosensor for the detection of Mycobacterium tuberculosis complex
}

Junfei Huang ${ }^{1}$, Ziyu Xiao ${ }^{1,2}$, Xinggui Yang ${ }^{1,2}$, Xu Chen $^{3}$, Xiaojuan Wang ${ }^{1,2}$, Yijiang Chen ${ }^{1}$, Wenlin Zheng ${ }^{1}$, Wei Chen ${ }^{1}$, Huijuan Chen ${ }^{1}$ and Shijun $\mathrm{Li}^{1,2^{*}}$

\begin{abstract}
Background: Tuberculosis (TB) is a serious chronic infectious disease caused by Mycobacterium tuberculosis complex (MTBC). Hence, the development of a novel, simple, rapid and sensitive method to detect MTBC is of great significance for the prevention and treatment of TB.

Results: In this study, multiple cross displacement amplification (MCDA) combined with a nanoparticle-based lateral flow biosensor (LFB) was developed to simultaneously detect two target genes (IS6110 and mpb64) of MTBC (MCDALFB). One suite of specific MCDA primers designed for the 156110 and mpb64 genes was validated using genomic DNA extracted from the reference strain H37Rv. The MCDA amplicons were analyzed using a real-time turbidimeter, colorimetric indicator (malachite green, MG) and LFBs. The optimal amplification temperature and time were confirmed, and the MCDA-LFB method established in the current report was evaluated by detecting various pathogens (i.e., reference strains, isolates and clinical sputum samples). The results showed that the two sets of MCDA primers targeting the 156110 and mpb64 genes could effectively detect MTBC strains. The optimal reaction conditions for the MCDA assay were determined to be $67^{\circ} \mathrm{C}$ for $35 \mathrm{~min}$. The MCDA assay limit of detection (LoD) was $100 \mathrm{fg}$ per reaction for pure genomic DNA. The specificity of the MCDA-LFB assay was 100\%, and there were no cross-reactions for non-MTBC strains. For sputum samples and MTBC strain detection, the positive rate of MCDA-LFB for the detection of MTBC strains was consistent with seminested automatic real-time PCR (Xpert MTB/RIF) and higher than acid-fast staining (AFS) and culture assays when used for sputum samples. The MCDA-LFB assay was a rapid tool, and the whole procedure for MCDA-LFB, including DNA template preparation, MCDA reaction and amplification product analysis, was completed within 70 min.
\end{abstract}

Conclusion: The MCDA-LFB assay targeting the 156110 and mpb64 genes is a simple, rapid, sensitive and reliable detection method, and it has potential significance for the prevention and treatment of TB.

Keywords: Mycobacterium tuberculosis complex, Multiple cross displacement amplification, Lateral flow biosensor, Detection, Diagnosis

*Correspondence: zjumedjun@163.com

2 Public Health School, Guizhou Medical University, Guiyang 550025,

Guizhou, People's Republic of China

Full list of author information is available at the end of the article

\section{Background}

Tuberculosis (TB) is a chronic infectious disease caused by Mycobacterium tuberculosis complex (MTBC), and the MTBC pathogens evolve M. tuberculosis, M. bovis, $M$. 
bovis Bacillus Calmette-Guerin (BCG), M. africanum, $M$. carinii, $M$. suricattae, $M$. orygis, $M$. microti, $M$. caprae, M. mungi, M. canettii, M. pinnipedii, and M. vole. In particular, M. tuberculosis, M. bovis and M. africanum are highly pathogenic bacteria [1-4]. TB seriously endangers human health and is a public health and social problem of global concern [5]. The World Health Organization (WHO) has listed TB as one of the major infectious diseases. In 2018, there were approximately 10 million new TB cases and 1.5 million TB-related deaths worldwide [5]. Approximately one-third of the world's people have been and/or are now infected with MTB, according to estimates from the World Health Organization [6, 7]. Thus, quick and accurate strategies for the detection and identification of MTBC strains are important for the prevention and treatment of TB. In general, the diagnosis methods for TB usually rely on conventional sputum smear microscopy (SSM) and culture identification of the organism (namely, mycobacterial cultivation identification and biochemical tests). However, deficiencies for the abovementioned conventional examination methods (including low sensitivity, time-consuming, and complicated operation steps) cannot meet the requirements for rapid and specific detection of MTBC. Hence, simple, fast, accurate and reliable detection methods are required for the detection of MTBC in laboratory diagnosis $[6,8]$. With the development of molecular techniques, polymerase chain reaction (PCR) and PCR-based assays (e.g., real-time PCR, nested PCR, and GeneXpert) have been widely used for the diagnosis of TB [9-12]. Although PCR and PCR-based assays have excellent sensitivity and reliability, the needs for specific instruments and/or reagents hinder their application in basic laboratories. To overcome the shortcomings of PCR techniques, isothermal amplification techniques, including loop-mediated isothermal amplification (LAMP), cross-priming isothermal amplification (CPA), and multiple cross displacement amplification (MCDA), were developed and applied for the diagnosis of TB [13-15]. In particular, the MCDA assay based on the isothermal strand-displacement polymerization reaction, which is a highly specific and sensitive detection technique, has been established and implemented in previous studies $[16,17]$. The target sequence has ten special primers spanning ten distinct regions and requires a constant temperature to react [16-18]. Real-time turbidity, agarose gel electrophoresis, colorimetric indicators and nanoparticle-based lateral flow biosensors (LFBs) were selected for MCDA product analysis, especially MCDA combined with nanoparticlebased LFB (MCDA-LFB), which makes product analysis simple and visual [15-19]. The MCDA technique was applied for the detection of Listeria monocytogenes [18], Salmonella spp. strains and Shigella spp. [19] and Vibrio parahaemolyticus [20]. Our team also created the MCDA method for Brucella spp. [17] and Neisseria meningitidis [16] detection successfully.

In this study, the MCDA-LFB method for MTBC detection was successfully established by our group. Two specific target genes, IS6110 and mpb64, were chosen for MTBC-MCDA detection, and 10 specific primers were designed for the targets. The MCDA products were analyzed using LFB, real-time turbidity and colorimetric indicators (malachite green, MG). Then, the MCDA reaction conditions, including amplification temperature and time, were optimized. Subsequently, the sensitivity and specificity of the MTBC-MCDA-LFB technique were tested, and then MCDA-LFB was applied to detect sputum specimens and MTBC strains from clinical samples.

\section{Results}

Confirmation and detection of MCDA products

The disposable lateral flow biosensor (LFB) consists of test Line 1 (TL1), test Line 2 (TL2) and a control line (CL). First, the $I S 6110$ gene (Fig. 1A) and mpb64 gene (Fig. 1B) were examined by MCDA amplification and detected with LFB and MG, respectively. Then, both target genes were detected at the same time (Fig. 1C). Genomic templates of the standard strain $M$. tuberculosis (H37Rv, ATCC 27294) were used for the MCDA assay.

\section{Optimization of the temperatures for the MTBC-MCDA assay}

To evaluate the optimum amplification temperature, $M$. tuberculosis (H37Rv, ATCC 27294) strain genomic templates were used as the positive controls at a level of $100 \mathrm{pg}$ per reaction, and the reactions were monitored by the real-time turbidity (LA-320C) method. Both the IS6110 gene and mpb64 gene were detected, and the effect was examined at fixed temperatures ranging from 63 to $70^{\circ} \mathrm{C}$ with $1^{\circ} \mathrm{C}$ intervals for MCDA amplification. The $M$. avium genomic templates were used as negative controls. According to Table 1 and Fig. 2, at $67^{\circ} \mathrm{C}$, both IS6110 and mpb64 gene amplification had the shortest time with higher turbidity and faster reaction times at 30 and $23 \mathrm{~min}$. Thus, an amplification temperature of $67^{\circ} \mathrm{C}$ was applied to perform the remaining experiments in the study.

\section{Optimization of reaction time for the MTBC-MCDA-LFB assay}

The four reaction times $(20,30,40$ and $50 \mathrm{~min})$ were tested at $67^{\circ} \mathrm{C}$ according to the standard MCDA conditions for the optimum time by the MTBC-MCDALFB assay during the reaction stage. The DNA level with $100 \mathrm{pg}$ of $M$. tuberculosis genomic templates per reaction was displayed by three visible-red lines (TL1, 


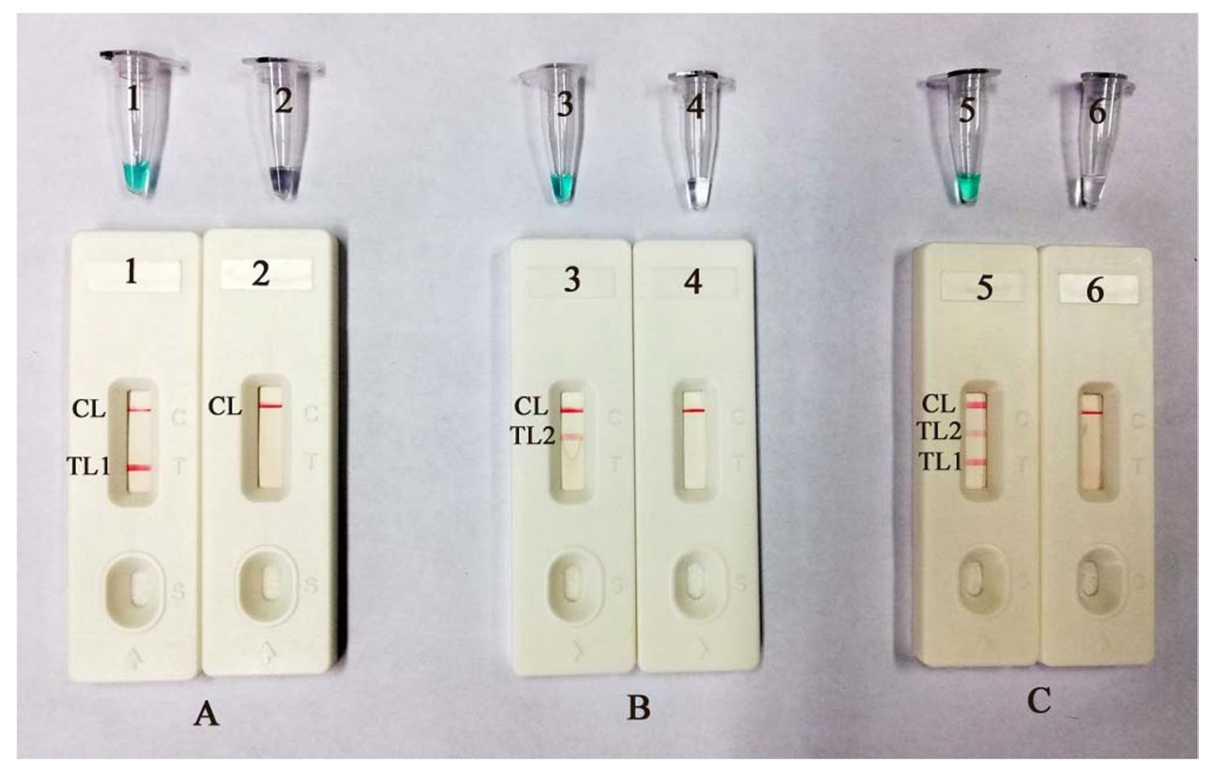

Fig. 1 Confirmation and detection of MTBC-MCDA products. A The LFB and MG methods were applied for 156110 gene amplification. The products of the MTBC-MCDA assay were visually analyzed by observation of TL1 and color change. Tube 1/biosensor 1: positive amplification of M. tuberculosis; Tube 2/biosensor 2: black control of DW. B The LFB and MG methods were applied for mpb64 gene amplification. The products of the MTBC-MCDA assay were visually analyzed by observation of TL2 and color change. Tube 3/biosensor 3: positive amplification of $M$. tuberculosis; Tube 4/biosensor 4: black control of DW. C The LFB and MG methods were applied for both 156110 and mpb64 gene amplification. The products of the MTBC-MCDA assay were visually analyzed by observation of TL1, TL2 and color change. Tube 5/biosensor 5: positive amplification of M. tuberculosis; Tube 6/biosensor 6: black control of DW

Table 1 Reaction temperature optimization for MTBC-MCDA primers

\begin{tabular}{|c|c|c|c|c|}
\hline \multirow{2}{*}{$\begin{array}{l}\text { Temperature } \\
\left({ }^{\circ} \mathrm{C}\right)\end{array}$} & \multicolumn{2}{|c|}{ IS6110 gene } & \multicolumn{2}{|c|}{ mpb64 gene } \\
\hline & $\begin{array}{l}\text { Peak of } \\
\text { turbidity }\end{array}$ & Time (min) & $\begin{array}{l}\text { Peak of } \\
\text { turbidity }\end{array}$ & Time (min) \\
\hline 63 & 0.16 & 34 & 0.18 & 29 \\
\hline 64 & 0.14 & 31 & 0.20 & 27 \\
\hline 65 & 0.17 & 32 & 0.21 & 26 \\
\hline 66 & 0.18 & 30 & 0.21 & 25 \\
\hline 67 & 0.18 & 30 & 0.22 & 23 \\
\hline 68 & 0.16 & 34 & 0.21 & 25 \\
\hline 69 & 0.15 & 38 & 0.20 & 25 \\
\hline 70 & 0.13 & 55 & 0.20 & 26 \\
\hline
\end{tabular}

The threshold of turbidity $>0.1$ was judged as positive for the MCDA reaction by a real-time turbidimeter

TL2 and CL) on the LFB. The earliest test lines were observed for both TL1 and TL2 when the amplification lasted for $30 \mathrm{~min}$ (the threshold of turbidity $>0.1$ ) (Fig. 3). To ensure adequate amplification, a reaction time of $35 \mathrm{~min}$ was recommended as a reasonable reaction time for the MTBC-MCDA-LFB assay in this research.

\section{Sensitivity of the MTBC-MCDA-LFB assay}

The genomic DNA templates of M. tuberculosis (H37Rv, ATCC 27294) were serially diluted (100 ng, $10 \mathrm{ng}, 1 \mathrm{ng}$, $100 \mathrm{pg}, 10 \mathrm{pg}, 1 \mathrm{pg}, 100 \mathrm{fg}, 10 \mathrm{fg}$ and $1 \mathrm{fg}$ per microliter) for MTBC-MCDA sensitivity analysis. The limiting dilution of M. tuberculosis genomic DNA was evaluated by MCDA detection. The single-MCDA-LFB assay limit of detection (LoD) targeting the $I S 6110$ gene (Fig. 4A) or mpb64 gene (Fig. 4B) was found to be $10 \mathrm{fg}$ per reaction. Double target genes were found to be $100 \mathrm{fg}$ per reaction (Fig. 4C). As expected, CL, TL1 and/or TL 2 were observed on the biosensor, displaying positive MCDA results for the IS6110 gene and/or mpb64 gene. Double distilled water (DW) was the template for the blank control. Moreover, the analytical sensitivity of MTBCMCDA with the biosensor was consistent with colorimetric indicator analysis.

\section{Specificity of the MTBC-MCDA-LFB assay}

Thirty-eight MTBC strains, 12 NTM strains and 10 other bacteria were used to determine the specificity of the MCDA-LFB assay. When the genomic DNA of the bacteria listed in Table 2 was used for MCDA-LFB detection, only the DNA of the MTBC strains tested positive. The genomic DNA of NTM and other bacterial strains tested negative by the MCDA-LFB assay. 


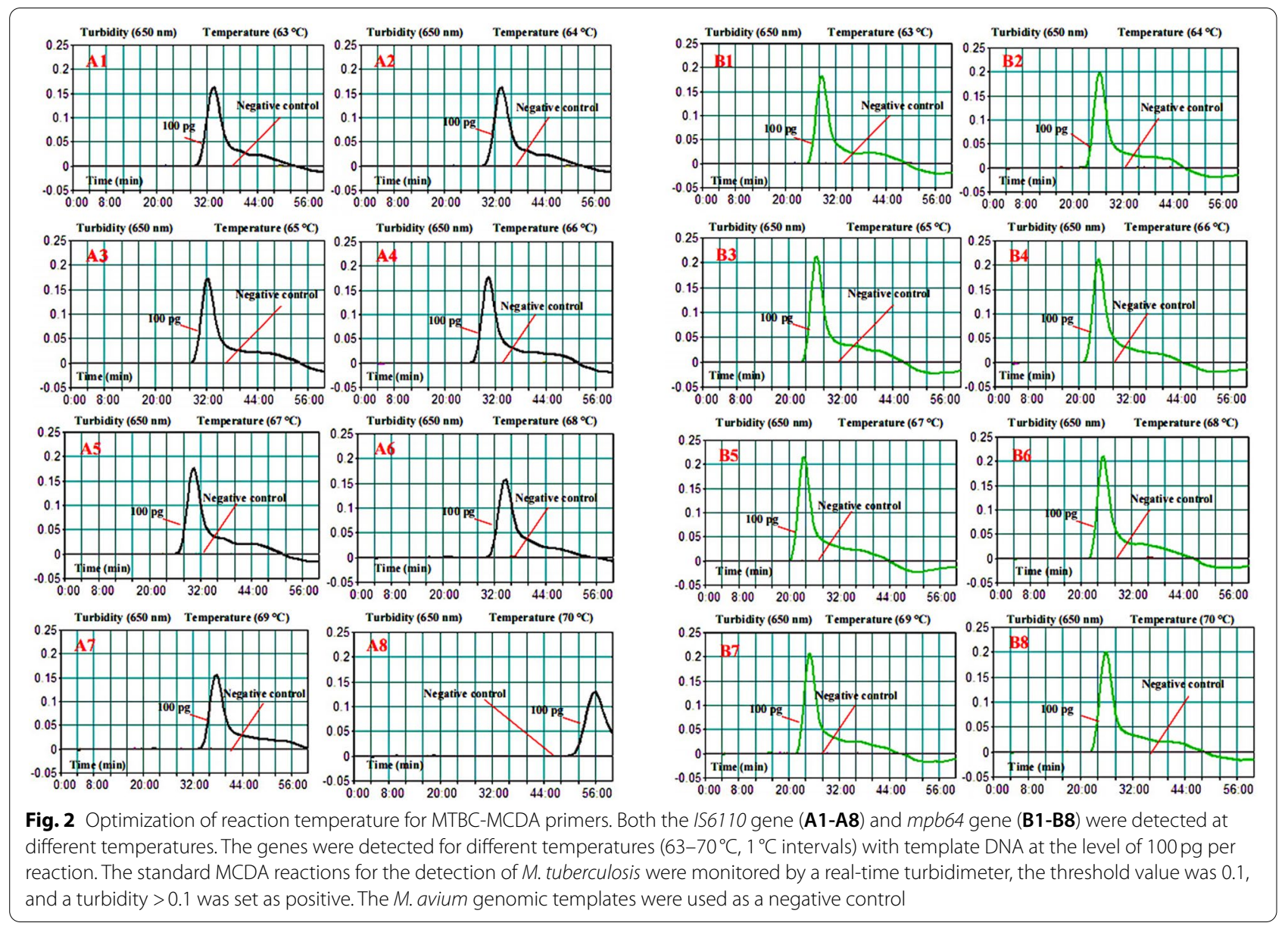

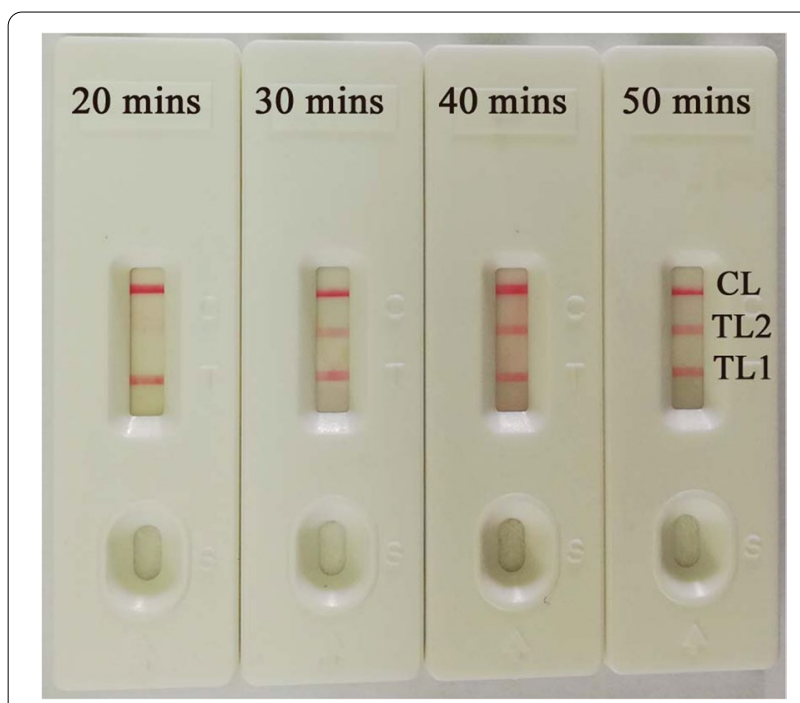

Fig. 3 The optimal duration of time required for the

MTBC-MCDA-LFB method. Four distinct reaction times (20, 30, 40, and $50 \mathrm{~min}$ ) were examined and compared at $67^{\circ} \mathrm{C}$. Biosensors represent DNA levels of $100 \mathrm{pg}$ of M. tuberculosis templates

\section{Application of the MTBC-MCDA-LFB assay for sputum samples}

Fifty-one sputum samples (provided by pulmonary hospital of Guiyang) were detected by acid-fast staining (AFS), conventional culture method, seminested automatic real-time PCR (Xpert MTB/RIF) and MCDALFB. The AFS results consisted of 26 positive samples and 25 negative samples, and the positive detection rate was 50.98\%. Additionally, the sputum samples were cultured, and the results showed that 35 samples were positive and 16 were negative after 8 weeks. The Xpert $\mathrm{MTB} / \mathrm{RIF}$ results indicated that 39 were positive and 12 were negative, and the rate of positive detection was $76.47 \%$. The MCDA-LFB results showed that 41 were positive and 10 were negative, and the rate of positive detection was $80.39 \%$ (Table 3 ).

\section{Discussion}

To date, TB remains a chronic infectious disease caused by MTBC members that seriously endangers human health $[1,5]$. Thus, convenient, rapid, sensitive and specific detection of MTBC is important for the prevention 
and treatment of $\mathrm{TB}$, especially the development of novel rapid detection techniques. However, conventional detection methods (i.e., SSM, mycobacterial cultivation identification and biochemical tests) usually cannot meet the needs of rapid detection in terms of the detection period and sensitivity $[6,8]$. PCR and PCR-based assay techniques displayed certain reliability and sensitivity in previous publications, but the requirements for PCR thermal cyclers and expensive reagents hindered their application in resource-poor areas [8]. Among the various rapid detection methods, MCDA, as a low-cost (the cost of a single MCDA reaction was estimated to be approximately 5 USD, and the LFB was 2.5 USD per test), highly sensitive and specific assay based on the LAMP technique [21], was established and applied for the detection of various pathogens (e.g., bacteria, viruses, and fungi) [21-23]. Currently, verification methods for MCDA amplicons have always been a major concern, especially for multiplex MCDA assays. Unfortunately, conventional methods (containing visual reagents, agarose gel electrophoresis and real-time turbidimetry) have difficulty verifying multiplex MCDA amplicons. Thus, LFBs based on nanoparticles were designed and utilized in our experiments. In this report, the MCDA technique combined with a nanoparticle-based LFB (MCDA-LFB) for rapid detection of MTBC was developed successfully.

In the current report, the IS6110 and mpb64 genes were chosen as specific molecular targets, and two sets of MCDA primers were designed. The IS6110 sequence, which belongs to a family of ISs of the IS3 category with high specificity, has been widely applied for MTBC-PCR assays [24]. However, some M. tuberculosis strains lacking the IS6110 gene were found in several clinical investigations $[25,26]$. Thus, the $m p b 64$ gene, which encodes the RD2 region of the MPB64 protein in the genome of M. tuberculosis [27], was introduced in our study, and it showed excellent specificity for MTBC strains in previous studies $[9,27]$. There is some evidence for the absence of the mpb64 gene in some substrains of Mycobacterium bovis BCG [28]. However, there are no reports of the absence of both the $I S 6110$ and mpb64 genes. In this study, we chose both the IS6110 gene and mpb64 gene as the target genes for MTBC to ensure its specificity. The
MCDA-LFB technique targeting the IS6110 and mpb64 genes was successfully established and applied to the detection of MTBC. As Table 2 shows, 38 MTBC strains, 12 NTM strains and 10 other bacterial strains were used to test the specificity of the MCDA-LFB assay. The specificity of the MCDA-LFB assay was 100\%, and there was no cross-reactivity with other pathogens (including NTM and nonmycobacterial strains).

In this research, the MCDA products were analyzed using the LFB, MG indicator and real-time turbidity methods. Comparing the above three validation methods, the LFBs were more convenient, reliable and visualized. They did not require special instruments or reagents $[16,17]$. In addition, LFB can detect both the IS6110 and mpb64 genes and allow them to be visualized in a single test at the same time. According to the results, at $67^{\circ} \mathrm{C}$, both $I S 6110$ and $m p b 64$ gene amplification had higher turbidity and shorter reaction times at $30 \mathrm{~min}$ and 23 min (Fig. 2 and Table 1). The LFB assay indicated that the earliest test lines were observed for both TL1 and TL2 when the amplification lasted for $30 \mathrm{~min}$ at $67^{\circ} \mathrm{C}$, but the TL2 line was light red (Fig. 3). Hence, to ensure adequate amplification, the conditions of amplification temperature and time were optimized for MCDA-LFB at $67^{\circ} \mathrm{C}$ for $35 \mathrm{~min}$ in this study. In addition, based on the $I S 6110$ and mpb64 genes, the MCDA assay LoD was $100 \mathrm{fg}$ of genomic DNA per reaction (Fig. 4). We found that the sensitivity of the single MCDA assay ( $10 \mathrm{fg}$ per reaction) was higher than that of the multiplex MCDA assay ( $100 \mathrm{fg}$ per reaction) in the sensitivity experiment. This phenomenon may be caused by the different concentrations of the single set of primers in the singlex and/ or multiplex MCDA reaction system (e.g., the CP1 of IS6110 primers was $2.4 \mu \mathrm{M}$ in the singlex MCDA system and 1.2 $\mu \mathrm{M}$ in the multiplex MCDA system) [29].

For sputum sample detection, Xpert MTB/RIF and MCDA-LFB had higher positive rates than the conventional culture and acid-fast staining assays (Table 3 ). While this is a possible reason for culture method that there were no living MTBC strains in the sputum samples, the Xpert MTB/RIF and/or MCDA-LFB could detect the nucleic acid of the target strains. In addition, the positive rates of the Xpert MTB/

\footnotetext{
(See figure on next page.)

Fig. 4 Sensitivity of the MCDA method using serially diluted genomic DNA extracted from M. tuberculosis (H37Rv, ATCC27294). A total of two detection techniques, including the lateral flow biosensor (LFB) and colorimetric indicator (MG) methods, were applied to analyze the amplification products. Serial dilutions of target templates were subjected to standard MCDA reactions. DNA levels of $100 \mathrm{ng}, 10 \mathrm{ng}, 1 \mathrm{ng}, 100 \mathrm{pg}, 10 \mathrm{pg}, 1 \mathrm{pg}$, $100 \mathrm{fg}, 10 \mathrm{fg}$ and $1 \mathrm{fg}$ per reaction. Double distilled water was the template for the blank control. A Singly, the 156110 gene at genomic DNA levels of $100 \mathrm{ng}$ per reaction, $10 \mathrm{ng}$ per reaction, $1 \mathrm{ng}$ per reaction, $100 \mathrm{pg}$ per reaction, $10 \mathrm{pg}$ per reaction, $1 \mathrm{pg}$ per reaction, $100 \mathrm{fg}$ per reaction and $10 \mathrm{fg}$ per reaction produced positive reactions. B The $\mathrm{mpb64}$ gene at genomic DNA levels of $100 \mathrm{ng}$ per reaction, $10 \mathrm{ng}$ per reaction, $1 \mathrm{ng}$ per reaction, $100 \mathrm{pg}$ per reaction, $10 \mathrm{pg}$ per reaction, $1 \mathrm{pg}$ per reaction, $100 \mathrm{fg}$ per reaction and $10 \mathrm{fg}$ per reaction produced positive reactions. C Both the IS6110 gene and mpb64 gene at genomic DNA levels of $100 \mathrm{ng}$ per reaction, $10 \mathrm{ng}$ per reaction, $1 \mathrm{ng}$ per reaction, $100 \mathrm{pg}$ per reaction, $10 \mathrm{pg}$ per reaction, $1 \mathrm{pg}$ per reaction and $100 \mathrm{fg}$ per reaction produced positive reactions
} 

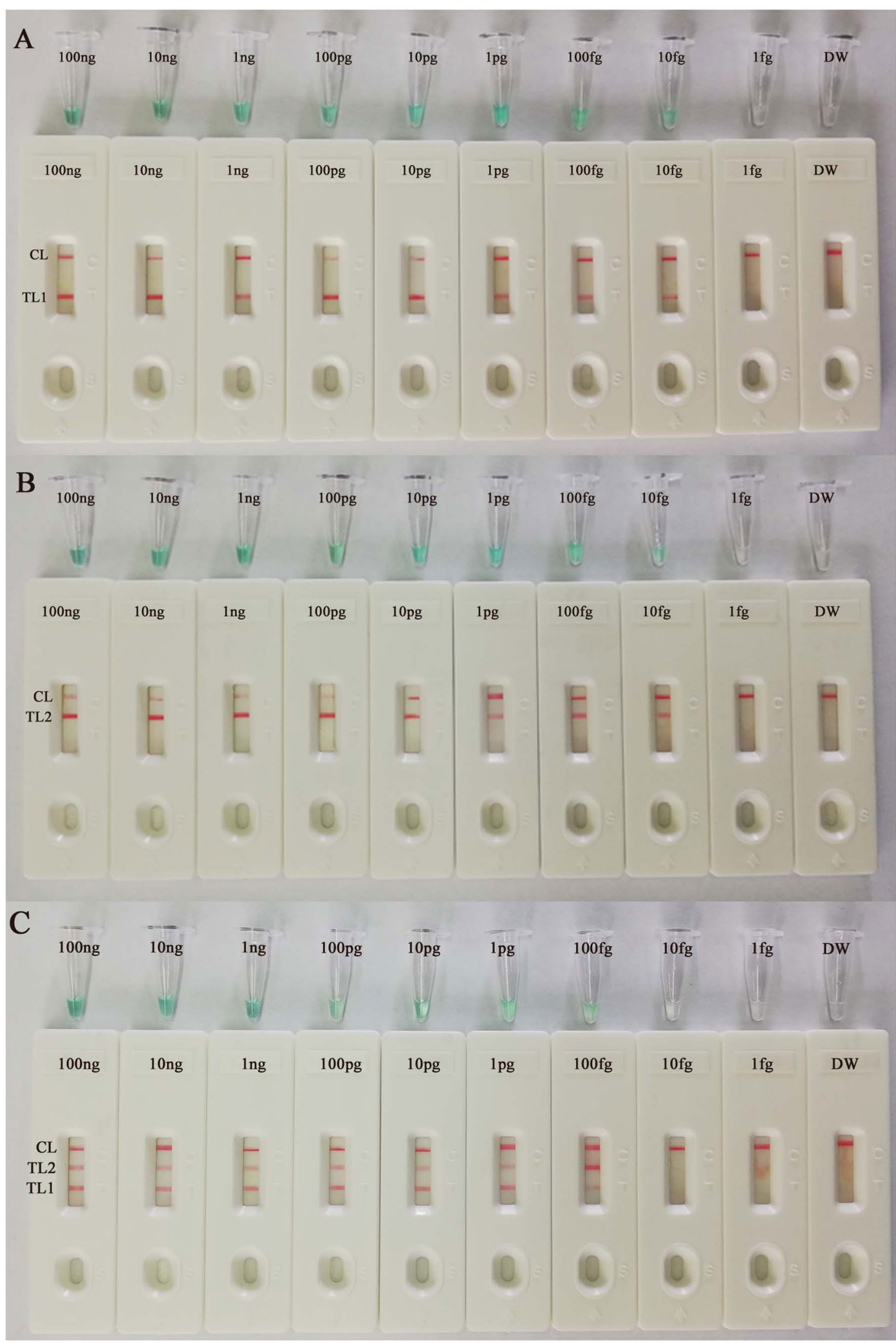

Fig. 4 (See legend on previous page.) 
Table 2 The details of the strains

\begin{tabular}{|c|c|c|c|}
\hline Bacteria & Strain no. (source of strains) & No. of strains & $\begin{array}{l}\text { MCDA- } \\
\text { LFB } \\
\text { result }\end{array}$ \\
\hline \multicolumn{4}{|l|}{ MTBC } \\
\hline M. tuberculosis & H37RvATCC27294 & 1 & $\mathbf{P}$ \\
\hline M. tuberculosis & Isolated strains (GZCDC) & 34 & $\mathbf{P}$ \\
\hline M. bovis & ATCC19210 & 1 & $\mathbf{P}$ \\
\hline M. africanum & ATCC25420 & 1 & $\mathbf{P}$ \\
\hline Bacillus Calmette-Guerin & Vaccine strain (GZCDC-BCG) & 1 & $\mathbf{P}$ \\
\hline \multicolumn{4}{|l|}{ NTM } \\
\hline M. aureus & ATCC23366 & 1 & $\mathbf{N}$ \\
\hline M. flavum & ATCC43999 & 1 & $\mathbf{N}$ \\
\hline M. avium & ATCC25291 & 1 & $\mathbf{N}$ \\
\hline M. marinum & ATACC927 & 1 & $\mathbf{N}$ \\
\hline M. abscess & ATCC19977 & 1 & $\mathbf{N}$ \\
\hline M. chelonae & ATCC14472 & 1 & $\mathbf{N}$ \\
\hline M. gordon & ATCC14470 & 1 & $\mathbf{N}$ \\
\hline M. phlei & ATCC11758 & 1 & $\mathrm{~N}$ \\
\hline M. nonchromogenic & ATCC19530 & 1 & $\mathbf{N}$ \\
\hline M.xenopi & ATCC19250 & 1 & $\mathbf{N}$ \\
\hline M. aichiense & ATCC27280 & 1 & $\mathbf{N}$ \\
\hline M.microti & ATCC19422 & 1 & $\mathbf{N}$ \\
\hline \multicolumn{4}{|l|}{ Other bacteria species } \\
\hline Enterococcus faecalis & Isolate strains (GZCDC) & 1 & $\mathbf{N}$ \\
\hline Salmonella & Isolate strains (GZCDC) & 1 & $\mathbf{N}$ \\
\hline Klebsiella pneumoniae & Isolate strains (GZCDC) & 1 & $\mathbf{N}$ \\
\hline Pseudomonas aeruginosa & Isolate strains (GZCDC) & 1 & $\mathbf{N}$ \\
\hline Staphylococcus aureus & Isolate strains (GZCDC) & 1 & $\mathbf{N}$ \\
\hline Escherichia coli & Isolate strains (GZCDC) & 1 & $\mathbf{N}$ \\
\hline Bacillus cereus & Isolate strains (GZCDC) & 1 & $\mathbf{N}$ \\
\hline Listeria monocytogenes & Isolate strains (GZCDC) & 1 & $\mathbf{N}$ \\
\hline Streptococcus pneumoniae & Isolate strains (GZCDC) & 1 & $\mathbf{N}$ \\
\hline Campylobacter jejuni & Isolate strains (GZCDC) & 1 & $\mathbf{N}$ \\
\hline
\end{tabular}

Abbreviations: MTBC Mycobacterium tuberculosis complex, NTM nontuberculous mycobacterium, ATCC American Type Culture Collection, GZCDC Guizhou Provincial Center for Disease Control and Prevention, $P$ positive, $N$ negative

RIF (76.47\%, 39/51) and MCDA-LFB assays (80.39\%, 41/51) for sputum specimens were similar (Table 3). Xpert MTB/RIF is an important tool for MTBC with

Table 3 Sputum samples and MTBC strain detection

\begin{tabular}{lll}
\hline Methods & \multicolumn{2}{l}{ Sputum samples $(\boldsymbol{N}=\mathbf{5 1})$} \\
\cline { 2 - 3 } & Positive & Negative \\
\hline Acid-fast staining & 26 & 25 \\
Culture & 35 & 16 \\
Gene-Xpert & 39 & 12 \\
MCDA-LFB & 41 & 10
\end{tabular}

Abbreviations: MCDA-LFB multiple cross displacement amplification with lateral flow biosensor rifampin (RIF) resistance [30], and the detection time is approximately $2.5 \mathrm{~h}$. Usually, Xpert MTB/RIF has a four-channel detection system, which means that this machine can test 4 samples at a time and is not suitable for the large-scale detection of MTB samples. Moreover, Xpert MTB/RIF needs to use the matching kit, which represents a high cost. However, the MCDALFB technique was a better choice for detecting MTBC with a large specimen size. Importantly, it is low cost (approximately 7.5 USD per test). Compared with the AFS method, combined microscopy detected MTB of sputum specimens, an approach that lacks sensitivity [31, 32] and takes approximately $2 \mathrm{~h}$, including fixing, dyeing, drying naturally and microscopy. Microscopy 
took more time as the samples increased. However, the MCDA-LFB technique can solve this problem well. The whole detection process was completed within $70 \mathrm{~min}$, including genomic template preparation (approximately $30 \mathrm{~min}$ ), MCDA reaction (approximately $35 \mathrm{~min}$ ) and LFB verification (approximately $1-2 \mathrm{~min}$ ), and the maximum detection quantity was 96 samples with a conventional PCR apparatus.

\section{Conclusions}

In this study, a reliable, rapid, visual, inexpensive and simple MCDA-LFB assay based on the IS6110 and mpb64 genes was successfully established for the detection of MTBC. This technique could identify the M. tuberculosis complex with high specificity, sensitivity, and rapidity, and it was a visual and simple assay of the MCDA products. Thus, the MTBC-MCDA-LFB method could be regarded as a useful technique for rapid and reliable identification of the $M$. tuberculosis complex in clinical samples. Moreover, the technique did not require complicated instruments and expensive reagents and could be used widely, especially in resource-limited areas of developing countries with a high TB burden.

\section{Methods}

\section{Reagents and apparatus}

DNA isothermal amplification kits, colorimetric indicators (malachite green, MG), and biotin-14-dCTP were purchased from Bei-Jing HaiTaiZhengYuan. Co., Ltd. (Beijing, China). DNA extraction kits (QIAamp DNA minikits; Qiagen, Hilden, Germany) were purchased from Qiagen (Beijing, China). The nanoparticle-based lateral flow biosensor (Disposable Lateral Flow Biosensor, LFB) was manufactured by Tian-Jin HuiDeXin Biotech. Co., Ltd. (Tianjin, China).

\section{Design of MCDA primers}

Two specific MTC-MCDA target genes, IS6110 (GenBank, Sequence ID: CP053903.1) and mpb64 (GenBank, Sequence ID: CP053903.1), were chosen. The 10 specific primers were designed by primer software PRIMER PREMIER 5.0 and Primer Explorer V4 [15]. In addition, FITC (fluorescein isothiocyanate) was labeled at the $5^{\prime}$ end of the $I 56110-\mathrm{C}^{*}$ primer, and digoxigenin (Dig) was labeled at the $5^{\prime}$ end of the mpb64-C1* primer. The details, including primer design, sequences and modifications, and locations in the expression sites of the IS6110 and mpb64 genes are listed in Table 4 and Fig. 5. All of the primer sequences were synthesized

Table 4 The details of primers for the IS6110 and mpb64 genes

\begin{tabular}{|c|c|c|c|}
\hline Genes & Primers name $^{a}$ & Sequences and modifications & Length \\
\hline \multirow[t]{10}{*}{ IS6110 } & IS6110-F1 & 5'-GGATGGTCGCAGAGATCC-3' & $18 \mathrm{nt}$ \\
\hline & IS6110-F2 & 5'-ATCGCGTTCGCCCTT-3' & $15 \mathrm{nt}$ \\
\hline & IS6110-CP1 & 5'-CGCGCAGCCAACACCAAGTAGCAGCACGATTCGGAGTG-3' & 38 mer \\
\hline & IS6110-CP2 & 5'-CCGGGACCACGACCGAAGACGCAATTCGGCGTTGTC-3' & 36 mer \\
\hline & IS6110-C1* & 5'-FITC-CGCGCAGCCAACACCAAGTAG-3' & $21 \mathrm{nt}$ \\
\hline & IS6110-C2 & 5'-CCGGGACCACGACCGAAGA-3' & $19 \mathrm{nt}$ \\
\hline & IS6110-D1 & 5'-ACCTCACTGATCGCTG-3' & $16 \mathrm{nt}$ \\
\hline & IS6110-D2 & 5'-ATCCGCTGAGCTGAAGC-3' & $17 \mathrm{nt}$ \\
\hline & IS6110-R1 & 5'-ACTTACGCACCGTCTC-3' & $16 \mathrm{nt}$ \\
\hline & IS6110-R2 & 5'-CAGGCGCAGGTCGATG-3' & $16 \mathrm{nt}$ \\
\hline \multirow[t]{10}{*}{$m p b 64$} & mpb64-F1 & 5'-CCCCGGGTTGAAGAAGA-3' & $17 \mathrm{nt}$ \\
\hline & mpb64-F2 & 5'-GCTCAAGGTCTACCAGAAC-3' & $19 \mathrm{nt}$ \\
\hline & mpb64-CP1 & 5'-ACAGGTATCGATAGCGCCGAATGCCCCGTCGTTCGTGACT-3' & 40 mer \\
\hline & mpb64-CP2 & 5'-TGCCACAGCGTGTCATAGGTACGACCACGTACAAGGC-3' & 37 mer \\
\hline & $\mathrm{mpb} 64-\mathrm{C} 1^{*}$ & 5'-Dig-ACAGGTATCGATAGCGCCGAATG-3' & $23 \mathrm{nt}$ \\
\hline & mpb64-C2 & 5'-TGCCACAGCGTGTCATAGGT-3' & $20 \mathrm{nt}$ \\
\hline & mpb64-D1 & 5'-CGGTGAATTATCAGAACTTC-3' & $20 \mathrm{nt}$ \\
\hline & mpb64-D2 & 5'-GCCTGGTCCCAATCGAA-3' & $17 \mathrm{nt}$ \\
\hline & mpb64-R1 & 5'-GTGAACTGAGCAAGCAGA-3' & $18 \mathrm{nt}$ \\
\hline & mpb64-R2 & 5'-ACAATGGGGAAGACGACT-3' & $18 \mathrm{nt}$ \\
\hline
\end{tabular}

Abbreviations: FITC fluorescein isothiocyanate, Dig digoxigenin, mer monomeric unit, $n t$ nucleotide

a IS6110-C1* ${ }^{*}$-labeled with FITC when used in the MCDA-LFB assay; mpb64-C1* $5^{\prime}$-labeled with Dig when used in the MCDA-LFB assay 


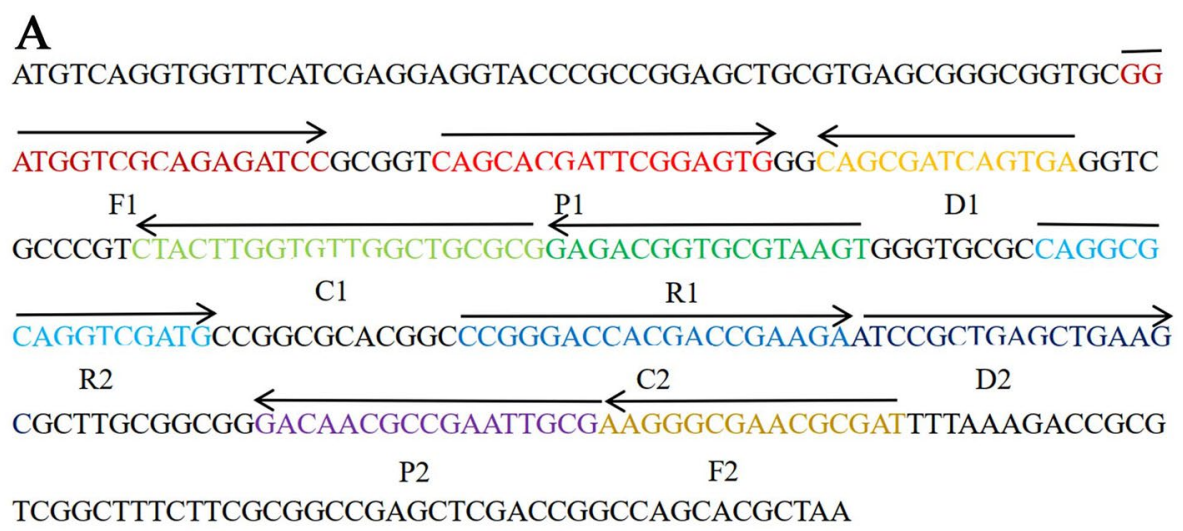

B

CTAGGCCAGCATCGAGTCGATCGCGGAACGTGGGACCAATACCTGGGTTGGGCCGGCT

GCTTCGGGCAGCAACTCCCCCGGGTTGAAGAAGAAAATCACCCCGTCGTTCGTGACT

GCGAAGTTCTGATAATTCACCGGGTCCAAGCCGGCATTCGGCGCTATCGATACCTGTTG

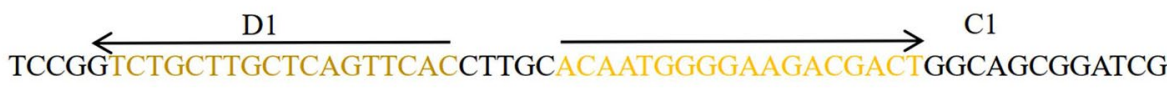

$\mathrm{R} 1 \longrightarrow \mathrm{R} 2$

GTGTCAGCCTGCCACAGCGTGTCATAGGT GATTGGCTTGCGATAGGCCTGGTCCCAATC

$\overrightarrow{\text { GAAGGCCTTGTACGTGGTCGTTGGGTGCGTGCCGCCGGCGTTCTGGTAGACCTTGAGC }}$

ACCACGGCCTGCGTACCACGCGGCGGTATCGCGGACTGGTATGTGGCCGAGGTGATAT

Fig. 5 Sequences, modifications and locations in the expression sites of the 156110 and $\mathrm{mpb64}$ genes. A Location and nucleotide sequence of the M. tuberculosis 156110 gene used to design the MCDA primers. B Location and nucleotide sequence of the M. tuberculosis mpb64 gene used to design the MCDA primers. All sequences of the primer sites are underlined. Right and left arrows indicate sense and complementary sequences that are used

and purified through Tian-Yi Biotech (Beijing, China) at HPLC purification grade.

\section{Bacterial strains and genomic DNA preparation}

There were 60 strains, including $38 \mathrm{MTBC}$ strains, 12 nontuberculous mycobacterium (NTM) strains and 10 other bacteria, and the details are shown in Table 2. The bacterial strains were stored in $10 \%(w / v)$ glycerol broth at $-80^{\circ} \mathrm{C}$ and then revived and cultured. According to the instruction book, the genomic templates were extracted from the cultured strains by the QIAamp DNA Mini Kit (Qiagen, Germantown, MD, USA). Sputum samples were treated with $4 \% \mathrm{NaOH}$ solution, and genomic templates were extracted by the kit. Then, the genomic templates were tested by ultraviolet spectrophotometry at A260/280 and stored at $-20^{\circ} \mathrm{C}$ before the templates were used.

\section{MCDA reaction and detection}

The suitability of two target genes for the MCDA primers was examined through MCDA reaction of the single IS6110 gene for the mpb64 gene. Then, both target genes were detected at the same time. Genomic templates of the standard strain M. tuberculosis (H37Rv, ATCC 27294) were used for the MCDA assay according to the DNA isothermal amplification kits. Briefly, the MCDA reaction mixtures contained $12.5 \mu \mathrm{l}$ of $2 \times$ Buffer, $1 \mu$ l of Bst 2.0 DNA polymerase (10 U), $1 \mu$ of biotin-14-dCTP, $1 \mu \mathrm{l}$ of malachite green, $2 \mu \mathrm{l}$ of genomic templates, $5.3 \mu \mathrm{l}$ of DW and $2.2 \mu \mathrm{l}$ of amplification primers or mixed primers containing $0.4 \mu \mathrm{M}$ each of displacement primers F1 and F2, $0.4 \mu \mathrm{M}$ each of amplification primers $\mathrm{C} 1$ * and $\mathrm{C} 2,1.2 \mu \mathrm{M}$ each of amplification primers R1, R2, D1 and D2, and $2.4 \mu \mathrm{M}$ each of cross primers CP1 and CP2 in a final volume of $25 \mu \mathrm{l}$. Then, 
they were reacted for $35 \mathrm{~min}$ at $67^{\circ} \mathrm{C}$. Three detection methods, including a real-time turbidimeter (LA-320C), disposable lateral flow biosensor (LFB) and colorimetric indicator (MG), were applied to detect the MCDA amplification products.

The threshold value (turbidity) was 0.1 , and a turbidity of $>0.1$ was regarded as positive amplification for the MCDA-LFB assay by using a real-time turbidimeter [16]. The LFB consisted of the sample pad, conjugate pad, NC membrane, and absorbent pad, which were laminated onto a plastic adhesive backing card. Then, the antiFITC Ab, anti-Dig Ab and biotin-BSA were sprayed onto the NC membrane for TL1, TL2 and CL, respectively [19]. The detection method of LFB involves depositing an aliquot $(1-2 \mu \mathrm{l})$ of MCDA amplification products on the sample pad of LFB and then depositing an aliquot of running buffer $(150-200 \mu \mathrm{l})$ on the sample pad of LFB. The amplicons (crimson red) were captured by specific antibodies (namely, anti-FITC $\mathrm{Ab}$, anti-Dig $\mathrm{Ab}$, and biotin-BSA) when flowing through the NC membrane. The red lines (i.e., CL, TL1 and/or TL2) were observed in the positive amplification, while only CL was red in the blank and negative controls. In addition, the color of positive amplification changed from blue to light blue, while the blank and negative controls were colorless by using MG indicators. In this study, DW was used as the template for the blank control, and the genomic DNA of $M$. avium was used as the template in the negative control.

\section{Optimizing the reaction temperature and time of the MCDA assay}

Both the IS6110 gene and mpb64 gene were detected at different temperatures by a real-time turbidimeter (LA320C). We examined the effect of different temperatures, from 63 to $70^{\circ} \mathrm{C}$, with $1{ }^{\circ} \mathrm{C}$ intervals for MCDA amplification. The $M$. avium genomic templates were used as a negative control. Subsequently, the two target genes were confirmed at different amplification reaction times, 20, 30, 40 and $50 \mathrm{~min}$, detected by LFBs and MG.

\section{Analytical sensitivity and specificity of MTBC-MCDA-LFB assay}

The genomic DNA templates of MTB (H37Rv, ATCC 27294 ) were serially diluted (100 ng- $1 \mathrm{fg}$ per microliter) for sensitivity analysis by MCDA-LFB detection for single genes and double genes. DW was the template for the blank control. Thirty-eight MTBC strains, 12 NTM strains and 10 other bacteria (Table 2) were used for the specificity assay. The genomic DNA from the strains was amplified by MCDA reactions and then assayed through LFB. The experiments were repeated at least two times.

\section{Practicability of the MTBC-MCDA-LFB assay for sputum samples}

Fifty-one sputum samples provided by the Pulmonary Hospital of Guiyang (China) were detected by AFS, conventional culture, Xpert MTB/RIF and MTBCMCDA-LFB methods. The AFS method was performed in our study according to previous publications [32]. Meanwhile, Xpert MTB/RIF and conventional culture methods were implemented according to the manufacturer's instructions. The MTBC-MCDA assay was carried out as described above, and its amplicons were validated by LFBs.

\section{Abbreviations}

MTBC: Mycobacterium tuberculosis complex; TB: Tuberculosis; MCDA: Multiple cross displacement amplification; LFB: Nanoparticle-based lateral flow biosensor; AFS: Acid-fast staining; WHO: World Health Organization; FITC: Fluorescein isothiocyanate; Dig: Digoxigenin. mer, monomeric unit; nt: Nucleotide; ATCC : American Type Culture Collection; LoD: Limit of detection; MG: Malachite green; GZCDC: Guizhou Provincial Center for Disease Control and Prevention; NTM: Nontuberculous mycobacterium; P: Positive; N: Negative; TL1: Test Line 1; TL2: Test Line 2; CL: Control line; DW: Double-distilled water; BC: Blank control; PC: Positive control; NC: Negative control.

\section{Acknowledgments}

We thank all of the medical workers in both the Public Health School, Guizhou Medical University and The Second Affiliated Hospital, Guizhou University of Traditional Chinese Medicine, for their cooperation in this study.

\section{Authors' contributions}

$J H$ and $S L$ conceived and designed the study. $J H, Z X$ and $S L$ participated in primer design. JH, ZX, XY, and XC contributed to all of the laboratory work. WX and YC contributed to the data collection. WZ, WC and HC performed the statistical analysis. JH and SL wrote the initial draft of the manuscript. JH and

$S L$ revised the manuscript. All authors read and approved the final manuscript.

\section{Funding}

This study was funded by grants from the Science and Technology Department of Guizhou Province (No. [2019]2822, [2019]1186), [2018]-5606 and [2018]-5767).

\section{Availability of data and materials}

The datasets used and/or analyzed during the current study are available from the corresponding author upon reasonable request. The raw sequence data reported in this paper came from GenBank, Sequence ID: CP053903.1, M. tuberculosis strain H37Rv_IC1chromosome (https://www.ncbi.nIm.nih.gov/ nuccore/CP053903.1?report=fasta).

\section{Declarations}

Ethics approval and consent to participate

The study was approved by the Human Ethics Committee of the Guizhou Provincial Center for Disease Control and Prevention and complied with the Declaration of Helsinki. All data/isolates were analyzed anonymously.

\section{Consent for publication \\ Not applicable.}

Competing interests

All of the authors declare that there are no competing interests in this article. 


\begin{abstract}
Author details
'Laboratory of Infectious Disease of Experimental Center, Guizhou Provincial Center for Disease Control and Prevention, 73 Bageyan Road, Guiyang 550004, Guizhou, People's Republic of China. ${ }^{2}$ Public Health School, Guizhou Medical University, Guiyang 550025, Guizhou, People's Republic of China. ${ }^{3} T$ The Second Affiliated Hospital, Guizhou University of Traditional Chinese Medicine, Guiyang, Guizhou 550003, People's Republic of China.
\end{abstract}

Received: 7 December 2020 Accepted: 24 September 2021 Published online: 04 October 2021

\section{References}

1. Fieweger RA, Wilburn KM. VanderVen BC: comparing the metabolic capabilities of Bacteria in the Mycobacterium tuberculosis complex. Microorganisms. 2019;7(6).

2. Cadmus SI, Jenkins AO, Godfroid J, Osinusi K, Adewole IF, Murphy RL, et al. Mycobacterium tuberculosis and Mycobacterium africanum in stools from children attending an immunization clinic in Ibadan, Nigeria. Int J Infect Dis. 2009;13(6):740-4.

3. Rodriguez-Campos S, Smith NH, Boniotti MB, Aranaz A. Overview and phylogeny of Mycobacterium tuberculosis complex organisms: implications for diagnostics and legislation of bovine tuberculosis. Res Vet Sci. 2014;97(Suppl):S5-S19.

4. Tientcheu LD, Koch A, Ndengane M, Andoseh G, Kampmann B, Wilkinson RJ. Immunological consequences of strain variation within the mycobacterium tuberculosis complex. Eur J Immunol. 2017;47(3):432-45.

5. Organization WHO: Global tuberculosis report 2019. Online at https:// www.who.int/teams/global-tuberculosis-programme/tb-reports/ global-report-2019.

6. Mwaba P, McNerney R, Grobusch MP, O'Grady J, Bates M, Kapata N, et al. Achieving STOP TB partnership goals: perspectives on development of new diagnostics, drugs and vaccines for tuberculosis. Tropical Med Int Health. 2011;16(7):819-27.

7. Zumla A, Atun R, Maeurer M, Mwaba P, Ma Z, O'Grady J, et al. Viewpoint: scientific dogmas, paradoxes and mysteries of latent Mycobacterium tuberculosis infection. Trop Med Int Health. 2011;16(1):79-83.

8. Schito M, Migliori GB, Fletcher HA, McNerney R, Centis R, D'Ambrosio L, et al. Perspectives on advances in tuberculosis diagnostics, drugs, and vaccines. Clin Infect Dis. 2015;61(suppl 3):S102-18.

9. Lekhak SP, Sharma L, Rajbhandari R, Rajbhandari P, Shrestha R, Pant B. Evaluation of multiplex PCR using MPB64 and 156110 primers for rapid diagnosis of tuberculous meningitis. Tuberculosis (Edinb). 2016:100:1-4.

10. Ji L, Jiang Y, Li G, Zhao X, Wan K. A real-time PCR assay based on a specific mutation of PstS1 gene for detection of M. bovis strains. Biologicals. 2020;64:23-7.

11. Sinha P, Prakash P, Patne SC, Anupurba S, Gupta S, Srivastava GN. Performance of nested multiplex PCR assay targeting MTP40 and IS6110 gene sequences for the diagnosis of tubercular lymphadenitis. J Microbiol. 2017:55(1):63-7.

12. Chakravorty S, Simmons AM, Rowneki M, Parmar H, Cao Y, Ryan J, et al. The new Xpert MTB/RIF ultra: improving detection of Mycobacterium tuberculosis and resistance to rifampin in an assay suitable for point-ofcare testing. MBio. 2017:8(4):e00812-17.

13. Joon D, Nimesh M, Varma-Basil M, Saluja D. Evaluation of improved 156110 LAMP assay for diagnosis of pulmonary and extra pulmonary tuberculosis. J Microbiol Methods. 2017;139:87-91.

14. Fang $\mathrm{R}$, Li X, Hu L, You Q, Li J, Wu J, et al. Cross-priming amplification for rapid detection of mycobacterium tuberculosis in sputum specimens. $J$ Clin Microbiol. 2009;47(3):845-7.

15. Wang Y, Wang Y, Ma A-J, Li D-X, Luo L-J, Liu D-X, et al. Rapid and sensitive isothermal detection of nucleic-acid sequence by multiple cross displacement amplification. Sci Rep. 2015;5:11902.

16. Li S, Liu C, Liu Y, Ma Q, Wang Y. Development of a multiple cross displacement amplification combined with nanoparticles-based biosensor assay to detect Neisseria meningitidis. Infect Drug Resist. 2019;12:2077-87.
17. Li S, Liu Y, Wang Y, Wang M, Liu C. Rapid detection of Brucella spp. and elimination of carryover using multiple cross displacement amplification coupled with nanoparticles-based lateral flow biosensor. Front Cell Infect Microbiol. 2019;9:78.

18. Wang Y, Li H, Wang Y, Li H, Luo L, Xu J, et al. Development of multiple cross displacement amplification label-based gold nanoparticles lateral flow biosensor for detection of listeria monocytogenes. Int J Nanomedicine. 2017;12:473-86.

19. Wang $Y$, Wang $Y$, Zhang $L, X u J$ J, Ye C. Visual and multiplex detection of nucleic acid sequence by multiple cross displacement amplification coupled with gold nanoparticle-based lateral flow biosensor. Sensors Actuators B Chem. 2017;241:1283-93.

20. Wang Y, Hui L, Li D, Li K, Wang Y, Xu J, et al. Multiple cross displacement amplification combined with gold nanoparticle-based lateral flow biosensor for detection of vibrio parahaemolyticus. Front Microbiol. 2016:7(770):2047.

21. Waterfield T, Fairley D, Lynn F, Blackwood B, Shields MD. A protocol for a systematic review of the diagnostic accuracy of loop-mediated-isothermal AMPlification (LAMP) in diagnosis of invasive meningococcal disease in children. Syst Rev. 2018;7(1):86.

22. Zhang W, Chen C, Cui J, Bai W, Zhou J. Application of loop-mediated isothermal amplification (LAMP) assay for the rapid diagnosis of pathogenic bacteria in clinical sputum specimens of acute exacerbation of COPD (AECOPD). Int J Clin Exp Med. 2015;8(5):7881-9.

23. Yang Q, Domesle KJ, Wang F, Ge B. Rapid detection of Salmonella in food and feed by coupling loop-mediated isothermal amplification with bioluminescent assay in real-time. BMC Microbiol. 2016;16(1):112.

24. Thierry D, Eisenach KD, Crawford JT, Bates JH, Gicquel B, Guesdon JL. IS6110, an IS-like element of Mycobacterium tuberculosis complex. Nucleic Acids Res. 1990;18(1)

25. Sharma K, Sharma M, Batra N, Sharma A, Dhillon MS. Diagnostic potential of multi-targeted LAMP (loop-mediated isothermal amplification) for osteoarticular tuberculosis. J Orthop Res. 2017:35(2):361-5.

26. Víctor Vinuesa RB, Briones ML, Clari MÁ, Cresencio V, Giménez E, Carmen Muñoz RO, et al. Performance of a highly sensitive mycobacterium tuberculosis complex real-time PCR assay for diagnosis of pulmonary tuberculosis in a low-prevalence setting: a prospective intervention study. J Clin Microbiol. 2018;56(5):e00116-8.

27. Tamada Y, Kanda S, Yoshidome A, Hayashi I, Miyake M, Nishiyama T. Diagnosis of active tuberculosis using MPB64, a specific antigen of Mycobacterium bovis. Microbiol Immunol. 2012;56(11):740-7.

28. Huayi LI, Ulstrup JC, Jonassen TO, Melby K, Nagai S, Harboe M. Evidence for absence of the MPB64 gene in some substrains of Mycobacterium bovis BCG. Infect Immun. 1993:61(5):1730-4.

29. Yang X, Huang J, Chen X, Xiao Z, Wang X, Chen Y, et al. Rapid and visual differentiation of mycobacterium tuberculosis from the mycobacterium tuberculosis complex using multiplex loop-mediated isothermal amplification coupled with a nanoparticle-based lateral flow biosensor. Front Microbiol. 2021:12:708658.

30. Boehme MD CC, Pamela Nabeta MD, Hillemann D, Nicol MP, Shubhada Shenai PD, Krapp F, et al. Rapid molecular detection of tuberculosis and rifampin resistance. N Engl J Med. 2010;363(11):1005-15.

31. Raj A, Singh N, Gupta KB, Chaudhary D, Yadav A, Chaudhary A, et al. Comparative evaluation of several gene targets for designing a multiplexPCR for an early diagnosis of Extrapulmonary tuberculosis. Yonsei Med J. 2016;57(1):88

32. Agrawal M, Bajaj A, Bhatia V, Dutt S. Comparative study of GeneXpert with ZN stain and culture in samples of suspected pulmonary tuberculosis. Clin Diagn Res. 2016;10(5):DC09-12.

\section{Publisher's Note}

Springer Nature remains neutral with regard to jurisdictional claims in published maps and institutional affiliations. 